\title{
A novel authigenic magnetite source for sedimentary magnetizations
}

\section{Geological background and sampling}

As one of the largest marginal basins in the western Pacific Ocean, the South China Sea is located at the junction of, and is controlled by, the complexly interacting Eurasian, Pacific, and India-Australian plates (Morley, 2012). The study site GMGS2-16 is situated in the Dongsha area

of the passive continental margin of the northern South China Sea. Well-developed bottom simulating reflectors, which indicate the base of the gas hydrate stability zone, are recognized widely in this area (McDonnell et al., 2000; Suess, 2005). During China's second gas hydrate drilling expedition (GMGS-2) in 2013, abundant gas hydrates were drilled in the Dongsha area (Sha et al., 2015) (Fig. 1). Core GMGS2-16 (871 m water depth) is one of five drill sites that yielded gas hydrates during the GMGS-2 drilling program (Fig. 1). The recovered sediments consist mainly of dark-green unconsolidated siliceous clayey silt and silty clay. Two gas hydrate layers were identified: (1) nodular and massive hydrates in the interval 15-30 m below seafloor (mbsf), and (2) massive hydrates in the interval 189-226 mbsf (Sha et al., 2015). After retrieval, sediment core GMGS2-16 was transferred temporarily to a cold room $\left(4^{\circ} \mathrm{C}\right)$ for storage. Samples were collected in a shore-based laboratory and were then stored frozen at $-20^{\circ} \mathrm{C}$ for geochemical and mineral analyses and at $4{ }^{\circ} \mathrm{C}$ for magnetic and other physical property analyses. 


\section{Magnetic mineral extraction from sediments}

After sampling, one aliquot of sediment was dried at $40^{\circ} \mathrm{C}$ in an oven for $24 \mathrm{~h}$ and was then sieved with distilled water using a $63 \mu \mathrm{m}$ sieve. Red-brown to black magnetic aggregates (Fig. DR1) were identified from this coarse fraction under a binocular microscope and were extracted with a rare earth hand magnet. The weight percent (wt.\%) of extracted magnetic aggregates in the coarse fraction was used to estimate the magnetic mineral content of magnetic extracts.

\section{In-situ X-ray diffraction (XRD) and electron probe microanalysis (EPMA) analyses}

Mineral compositions were identified using a Rigaku Rapid II XRD system (Mo Ka radiation) at the School of Marine Sciences, Sun Yat-sen University. Analyzed magnetic extracts were pasted to capillaries and were loaded onto the sample stage with centers aligned to the X-ray beam. Samples were set to spin during analysis and beam conditions were set to $50 \mathrm{kV}$ and $30 \mathrm{~mA}$ at room temperature. Diffraction patterns were collected by the image plate and were transformed into $2 \theta$-intensity profiles using the Rigaku 2DP software. Major mineral phases were identified using PDXL2 software. Chemical composition analysis of magnetically extracted aggregates was performed using a JEOL JXA-8800R EPMA system with wavelength dispersive X-ray spectroscopy at the Instrumental Analysis and Research Center, Sun Yat-sen University. 


\section{Scanning electron microscope (SEM) and transmission electron microscope (TEM) analyses}

Magnetic extracts were examined with a $\Sigma$ IGMA field emission SEM after carbon coating at the

School of Earth Science and Engineering, Sun Yat-sen University. Selected magnetic extracts were

loaded in a carbon-laced double-layer copper grid for TEM observations. Selected area electron diffraction (SAED) patterns and scanning TEM (STEM) and high-resolution TEM (HRTEM) images were obtained with a Thermo Scientific Talos F200S SEM/TEM at the Guangzhou Institute of Geochemistry, Chinese Academy of Sciences.

\section{Magnetic susceptibility $(\chi)$ and first-order reversal curve (FORC) measurements}

Magnetic measurements were performed on freeze-dried bulk samples. Sedimentary $\chi$ was measured at $976 \mathrm{~Hz}$ in a $200 \mathrm{~A} / \mathrm{m}$ field with an AGICO MFK1-FA Kappabridge system. FORCs were measured at $0.2 \mathrm{mT}$ increments with $0.5 \mathrm{~s}$ averaging time. All magnetic measurements were made at the School of Earth Sciences and Engineering, Sun Yat-sen University. 

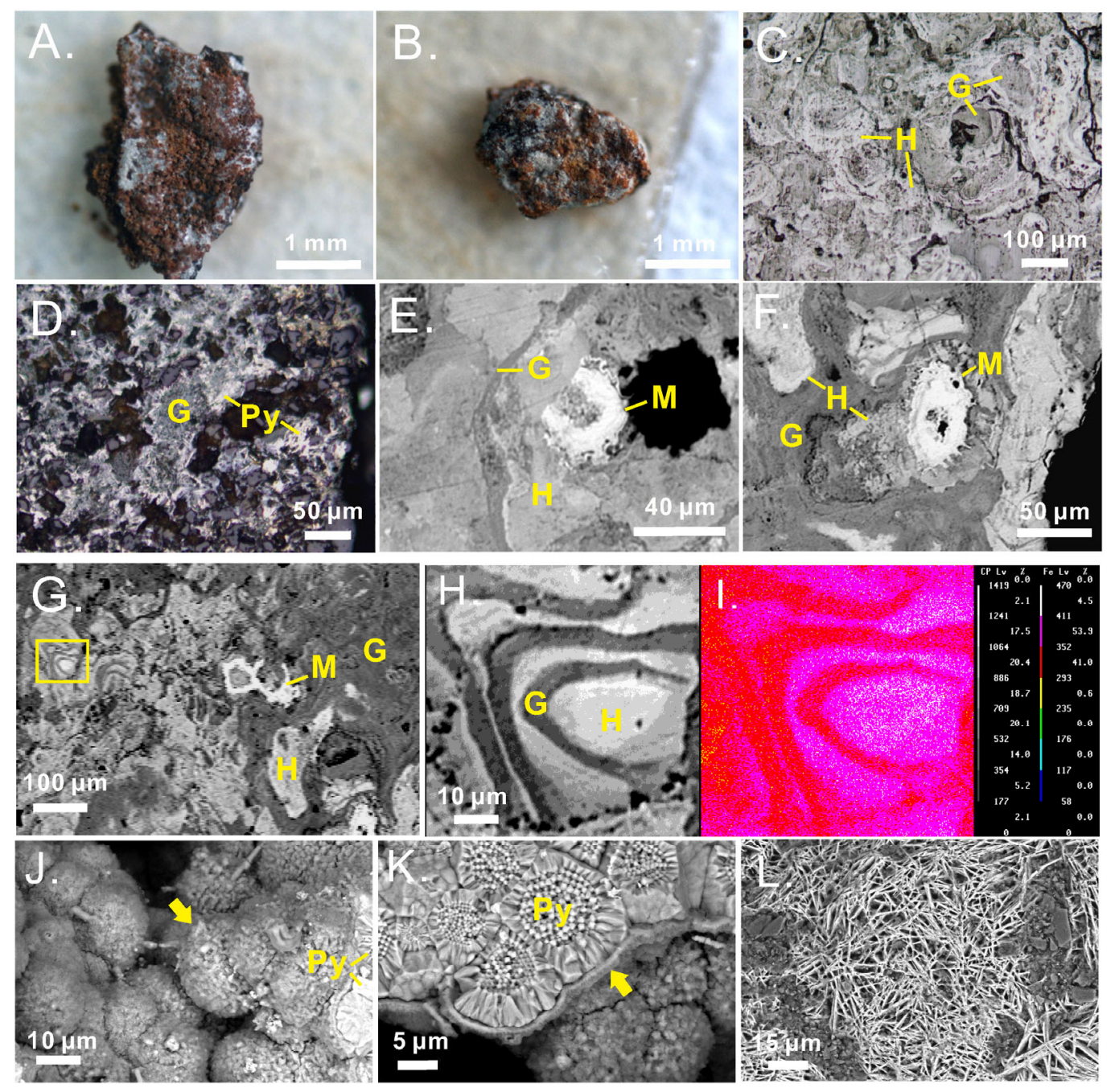

Fig. DR1. Typical morphologies and structures of iron (oxyhydr)oxides in sediments of core

GMGS2-16. (A, B) Typical magnetically extracted aggregates. (C, D) Reflected-light photograph

of a polished thin section that reveals a paragenetic sequence of hematite $(\mathrm{H})$, and goethite $(\mathrm{G})$ and pyrite (Py) in a magnetic extract. (E-G) Backscattered electron image of polished thin sections that reveal a paragenetic sequence of magnetite $(M)$, hematite $(H)$, and goethite $(\mathrm{G})$ in magnetic extracts. (H, I) EPMA elemental $(\mathrm{Fe})$ maps of goethite $(\mathrm{G})$ and hematite $(\mathrm{H})$ bands. $(\mathbf{H})$ Enlarged image of the area in the yellow rectangle in $(\mathbf{G}) .(\mathbf{J}, \mathbf{L})$ SEM images of secondary amorphous iron 
(oxyhydr)oxides encrusted on framboidal pyrite. Yellow arrows are iron (oxyhydr)oxides. (L)

Plate-like iron (oxyhydr)oxide aggregate in a magnetic extract.
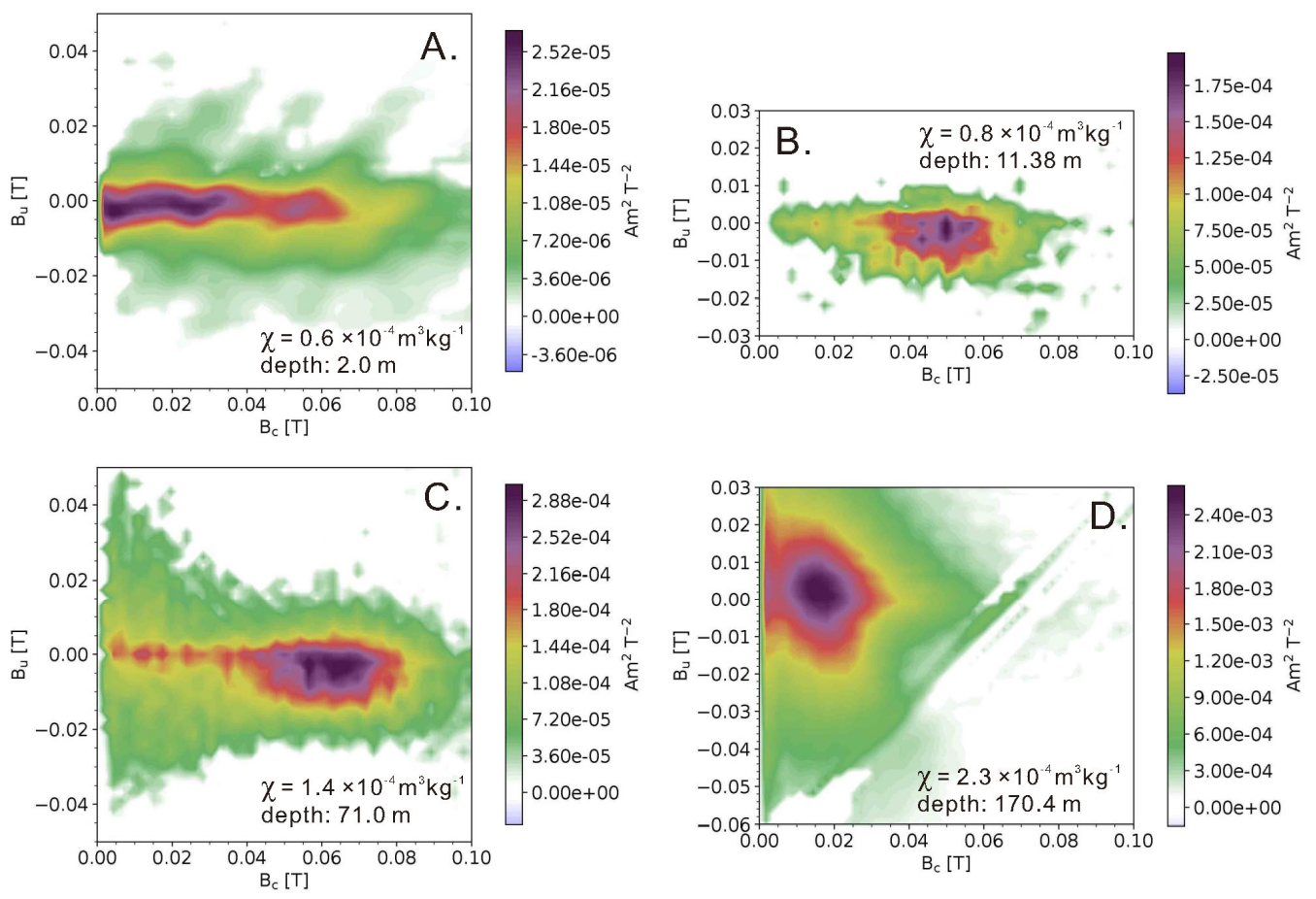

Fig. DR2. Further first-order reversal curve (FORC) diagrams for typical bulk sediments

with low magnetic susceptibility $(\chi)$ from different depths. The FORC diagrams in (A-C) reveal

a magnetostatically interacting medium-coercivity SD component, which is typical of

greigite-bearing sediments from sulfidic and methanic diagenetic environments (Roberts et al.,

2018). The FORC diagram in (D) is typical of the magnetostatically interacting stable SD or vortex

state magnetite assemblages illustrated in Fig. 2E2-4 (see Roberts et al. (2017) for interpretation of

vortex state signals). FORC diagrams were processed using the FORCsensei algorithm, which was

used to search 1350 FORC models using all combinations of VARIFORC smoothing parameters 
(Egli, 2013) to produce optimal FORC distributions in which noise is smoothed without over-smoothing the underlying signal (https://forcaist.github.io/FORCaist.github.io/). Optimal VARIFORC parameters for each diagram in this figure are: (A) $s_{\mathrm{c}, 0}=7, s_{\mathrm{c}, 1}=7, s_{\mathrm{u}, 0}=4, s_{\mathrm{u}, 1}=5, \lambda$ $=0.08$, and $\psi=0.32 ;$ (B) $s_{\mathrm{c}, 0}=2, s_{\mathrm{c}, 1}=2, s_{\mathrm{u}, 0}=2, s_{\mathrm{u}, 1}=2, \lambda=0.00$, and $\psi=0.24 ;(\mathrm{C}) s_{\mathrm{c}, 0}=2, s_{\mathrm{c}, 1}$ $=2, s_{\mathrm{u}, 0}=2, s_{\mathrm{u}, 1}=3, \lambda=0.04$, and $\psi=0.28$; and (D) $s_{\mathrm{c}, 0}=2, s_{\mathrm{c}, 1}=2, s_{\mathrm{u}, 0}=2, s_{\mathrm{u}, 1}=2, \lambda=0.04$, and $\psi=0.71$. Optimal VARIFORC parameters for each diagram for Fig. $2 \mathrm{E}$ are: $(1) s_{\mathrm{c}, 0}=4, s_{\mathrm{c}, 1}=4$, $s_{\mathrm{u}, 0}=2, s_{\mathrm{u}, 1}=3, \lambda=0.08$, and $\psi=0.25 ;(2) s_{\mathrm{c}, 0}=3, s_{\mathrm{c}, 1}=3, s_{\mathrm{u}, 0}=2, s_{\mathrm{u}, 1}=3, \lambda=0.08$, and $\psi=0.71$; (3) $s_{\mathrm{c}, 0}=2, s_{\mathrm{c}, 1}=2, s_{\mathrm{u}, 0}=2, s_{\mathrm{u}, 1}=2, \lambda=0.08$, and $\psi=0.53$; and (4) $s_{\mathrm{c}, 0}=2, s_{\mathrm{c}, 1}=2, s_{\mathrm{u}, 0}=3, s_{\mathrm{u}, 1}=$ $3, \lambda=0.12$, and $\psi=0.64$. 


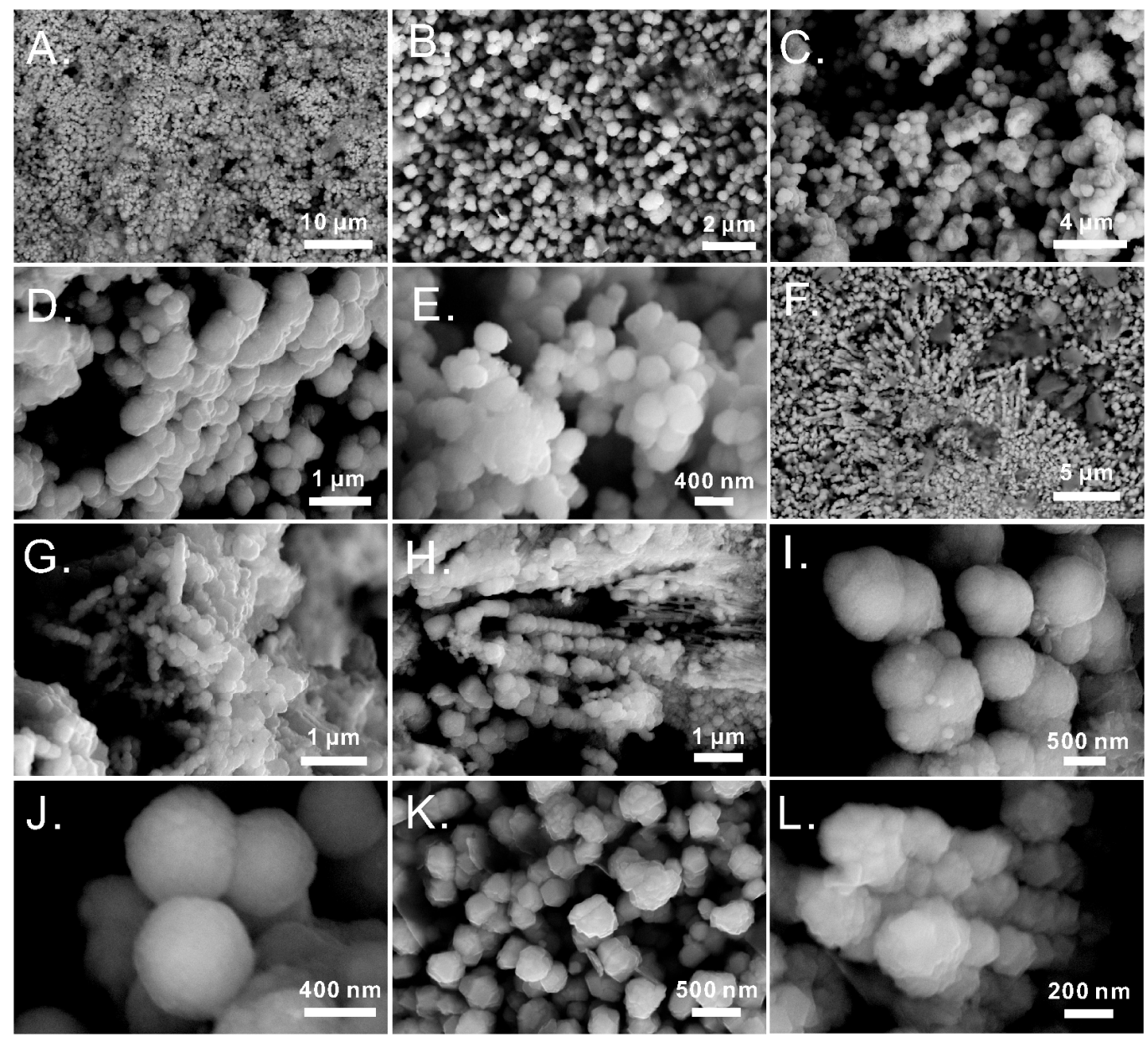

Fig. DR3. Further scanning electron microscope images of nanometer-scale magnetite aggregates in core GMGS2-16. (A-E) Magnetite particle aggregates. (F-H) Magnetite particle alignments. (I, J) Spherical particles. (K, L) Clustered euhedral crystals. 


\section{References Cited}

Egli, R., 2013, VARIFORC: An optimized protocol for calculating non-regular first-order reversal curve (FORC) diagrams: Global and Planetary Change, v. 110, p. 302-320, doi:10.1016/j.gloplacha.2013.08.003.

McDonnell, S.L., Max, M.D., Cherkis, N.Z., and Czarnecki, M.F., 2000. Tectono-sedimentary controls on the likelihood of gas hydrate occurrence near Taiwan: Marine and Petroleum Geology, v. 17, p. 929-936, doi:10.1016/S0264-8172(00)00023-4.

Morley, C.K., 2012. Late Cretaceous-Early Palaeogene tectonic development of SE Asia: Earth-Science Reviews, v. 115, p. 37-75, doi:10.1016/j.earscirev.2012.08.002.

Roberts, A.P., Almeida, T.P., Church, N.S., Harrison, R.J., Heslop, D., Li, Y., Li, J., Muxworthy, A.R., Williams, W., and Zhao, X., 2017, Resolving the origin of pseudo-single domain magnetic behavior: Journal of Geophysical Research: Solid Earth, v. 122, p. 9534-9558, doi:10.1002/2017JB014860.

Roberts, A.P., Zhao, X., Harrison, R.J., Heslop, D., Muxworthy, A.R., Rowan, C.J., Larrasoaña, J.C., and Florindo, F., 2018, Signatures of reductive magnetic mineral diagenesis from unmixing of first-order reversal curves: Journal of Geophysical Research: Solid Earth, v. 123, p. 4500-4522, doi:10.1029/2018JB015706.

Sha, Z., Liang, J., Zhang, G., Yang, S., Lu, J., Zhang, Z., McConnell, D.R., and Humphrey, G., 
2015. A seepage gas hydrate system in northern South China Sea: seismic and well log interpretations: Marine Geology, v. 366, p. 69-78, doi:10.1016/j.margeo.2015.04.006.

Suess, E., 2005. RV SONNE Cruise Report SO 177, Sino-German Cooperative Project, South

China Sea Continental Margin: Geological Methane Budget and Environmental Effects of Methane Emissions and Gas hydrates. IFM-GEOMAR Reports. 